\title{
The Bordeaux and Valinhos CCD meridian circles
}

\author{
B. Viateau ${ }^{1,2}$, Y. Réquième ${ }^{1}$, J.F. Le Campion ${ }^{1,2}$, P. Benevides-Soares ${ }^{2,1}$, R. Teixeira ${ }^{2,1}$, \\ G. Montignac $^{1,2}$, J.M. Mazurier ${ }^{1}$, W. Monteiro ${ }^{2}$, F. Bosq $^{1,2}$, F. Chauvet $^{1,2}$, J. Colin ${ }^{1}$, G. Daigne ${ }^{1}$, \\ J.M. Desbats ${ }^{1,2}$, T.P. Dominici ${ }^{2}$, J.P. Périé ${ }^{1}$, J. Raffaelli ${ }^{2}$, and M. Rapaport ${ }^{1}$ \\ 1 Observatoire de Bordeaux, UMR 5804, CNRS, BP. 89, F-33270 Floirac, France \\ 2 IAG-USP, Departamento de Astronomia, Caixa Postal 3386, 01060-970 São Paulo, Brazil
}

Received July 8; accepted July 27, 1998

\begin{abstract}
A first CCD $512 \times 512$ camera working in scan mode (declination field $14^{\prime}$ ) was mounted in 1994 on the Bordeaux CCD meridian circle. After a testing period, this camera was installed on the Valinhos CCD meridian circle (near São Paulo, Brazil), as part of a collaboration between Bordeaux Observatory and the Instituto Astronomico e Geofisico of São Paulo. A second improved CCD $1024 \times 1024$ camera, with a declination field of $28^{\prime}$, was installed on the Bordeaux instrument in June 1996. The mean internal precision of a single observation is about $0.04^{\prime \prime}$ in both coordinates for $9 \leq V \leq 14$. In the same magnitude range, magnitudes can also be obtained with an internal precision of about $0.05 \mathrm{mag}$. Both instruments can participate efficiently in extending the HipparcosTycho frame, during the next decade. Among other duties, the Bordeaux CCD meridian circle is being used since January 1997 for completing the Méridien 2000 project. The characteristics of both instruments and some results obtained with them are presented in this paper.
\end{abstract}

Key words: astrometry - reference systems instrumentation: miscellaneous

\section{Introduction}

The success of the European space mission HipparcosTycho, in the preparation of which the automatic meridian circle of Bordeaux Observatory participated from 1983 to 1988 , has raised the question of the future of the meridian circles. Availability of the Hipparcos optical reference frame with an accuracy at the milliarcsec level makes classical visual meridian instruments definitively obsolete. Even several automatic meridian circles have now been closed down: their limiting magnitude was too small and

$\overline{\text { Send offprint requests to }}$ : B. Viateau their observing rate too slow to effectively meet the needs of modern astrometry.

However from 1988 onwards, it was clearly demonstrated at Flagstaff (Stone 1993; Stone et al. 1996) that a CCD detector mounted at the focus of a meridian circle and working in drift scan mode could give a positional internal error of less than 50 mas up to $V=15-16$. Bordeaux Observatory and the Instituto Astronomico e Geofisico of São Paulo began a collaboration a few years later and decided to explore this new means with differential reductions using the Tycho catalogue as reference upon its release.

A first $512 \times 512$ pixel CCD drift scan camera with a $19 \mu \mathrm{m}$ pixel size was built at Bordeaux Observatory and mounted on the Bordeaux meridian circle in March 1994. After a testing period, this camera was moved onto the meridian circle of Valinhos Observatory in May 1995. A second larger camera $(1024 \times 1024)$ was mounted on the Bordeaux instrument in June 1996.

Réquième et al. (1997) briefly presented the work carried out with both meridian circles since they had been fitted with a CCD detector. The instruments, the methods used for image processing and reduction of the data are now described in more detail in the present paper. Some typical results are also shown, as well as the main ongoing programmes and projects for both instruments.

\section{The Bordeaux and Valinhos CCD drift scan cameras}

In drift scan mode, the rows of the CCD detector are parallel to the diurnal motion of stars, and electric charges are moved from each column of the detector to the next one at a rate matching precisely the rate of transit of the star image calculated for the declination of the centre of the field. Charges accumulated during the integration period, which is the time spent by the stars in front of the CCD detector, are collected by quickly emptying the last column of pixels during each charge transfer. Sky strips 
Table 1. Main features of the Valinhos and Bordeaux meridian circles and CCD detectors

\begin{tabular}{lccl}
\hline & Valinhos & Bordeaux & unit \\
\hline longitude west & $46^{\circ} 58^{\prime} 3^{\prime \prime}$ & $0^{\circ} 31^{\prime} 39^{\prime \prime}$ & \\
latitude & $-23^{\circ} 0^{\prime} 6^{\prime \prime}$ & $44^{\circ} 50^{\prime} 7^{\prime \prime}$ & \\
\hline diameter of front lens & 190 & 202 & $\mathrm{~mm}$ \\
focal length & 2590 & 2368 & $\mathrm{~mm}$ \\
\hline type of detector & Thomson $7895 \mathrm{M}$ & Thomson $7896 \mathrm{M}$ & \\
number of pixels & $512 \times 512$ & $1024 \times 1024$ & \\
pixel size & 19 & 19 & $\mu \mathrm{m}$ \\
pixel field & 1.51 & 1.65 & $\operatorname{arcsec}$ \\
declination field & 13 & 28 & $\operatorname{arcmin}$ \\
equivalent exposure time & $51 / \cos \delta$ & $112 / \cos \delta$ & seconds \\
magnitude range & $8.5 \leq V \leq 16$ & $8.5 \leq V \leq 16$ & \\
\hline
\end{tabular}

which are narrow in declination $\left(13^{\prime}\right.$ in Valinhos and $28^{\prime}$ in Bordeaux), and wide in right ascension, can be numerically reconstructed, up to several hours.

Basic features of the Valinhos and Bordeaux instruments and cameras are given in Table 1. Both cameras work in MPP (multi-pinned phase) mode and are cooled down to $-40^{\circ} \mathrm{C}$ by a two-stage thermoelectric Peltier module and water circulation. The overall dark noise of the CCD detector is about $65 \mathrm{e}^{-} / \mathrm{s}$. Charge transfer at the sidereal rate is controlled by a rubidium clock. The signal is converted by a 16-bit AD1377 converter. The observed star field is continuously displayed in real time. In order to reduce saturation effects for bright stars, an antiblooming circuit, producing electrical pulses on one of the 4 phases of the CCD detector during the integration period, is used. Software for the control of the instruments was developed at Bordeaux Observatory and is the same for both meridian circles.

The CCD detector covers a wide range of wavelengths (Fig. 1). On both instruments, filters are used to limit the spectral bandpass to values close to the focal minimum of the lens, for which changes in chromatic refraction are not too large. Filters GG495 + BG38 were selected, giving a spectral bandwidth of $520-680 \mathrm{~nm}$ and a mean wavelength of $605 \mathrm{~nm}$. With these filters, chromatic corrections, with respect to stars of spectral type K3, vary from $-0.04^{\prime \prime} \tan (z)$ for B0 stars to $0.04^{\prime \prime} \tan (z)$ for M 3 stars, where $z$ is the zenith distance.

\section{Image processing}

The purpose of the image processing is to search in the observed field for all the objects which may be detected, in order to identify them and to measure their positions and their fluxes.

Two axes $O x$ and $O y$, where $O$ is the origin of the field, $x$ is along the movement of the strip, and $y$ is perpendicular to $x$, determine the frame of reference of the observed field. Origin $O$ corresponds to the beginning of the strip in $x$ and to the centre of the strip in $y$. The columns in $y$

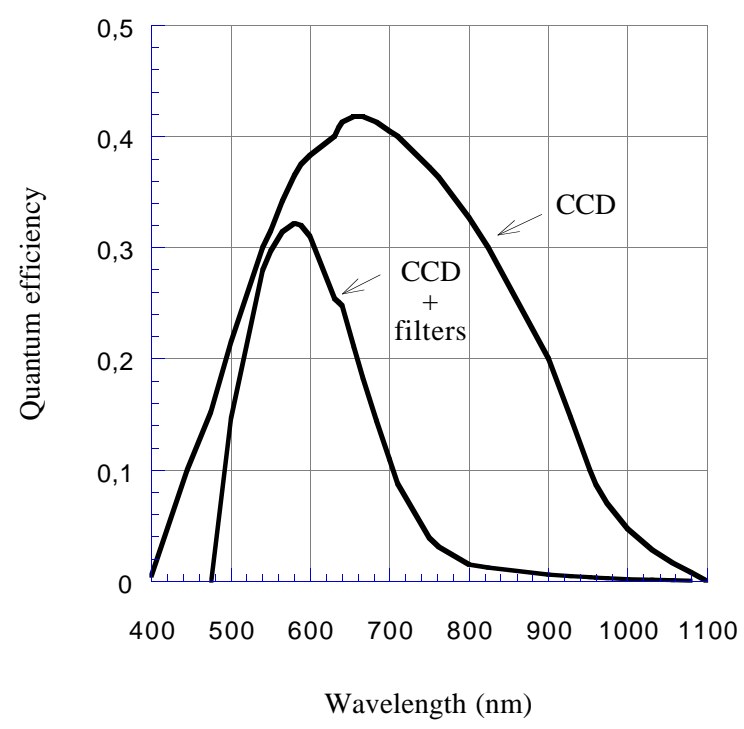

Fig. 1. Spectral response of the CCD detector, as given by the manufacturer

which make up the field are processed one by one in the order of their arrival.

For each column, image processing is achieved in 3 steps:

- extraction of the sky background;

- detection of the objects present in the field and their possible identification by comparison with a reference catalogue;

- measurement of the position and the flux of each detected object.

\subsection{Extraction of the sky background}

The extraction of the sky background is made either by fitting a polynomial of a degree between 0 and 3 on each column in $y$, or by using a median filter when the field has strong gradients of flux (e.g. near the Moon, observations of planetary satellites, presence of condensation or hoarfrost, ...). In most cases, there is no significant gradient 
of flux and the results obtained with both methods are rather similar.

\subsubsection{Polynomial fitting}

A polynomial is fitted on the flux received on each pixel of the column by least-squares and is substracted from the sky background. Each pixel gives the equation:

$$
\operatorname{flux}(i)=a\left[+b i\left[+c i^{2}\left[+d i^{3}\right]\right]\right]
$$

where $i$ is the number of the pixel in the column, $a, b, c, d$ are the unknowns of the system and brackets indicate optional terms which depend on the degree of the polynomial. Pixels with residuals greater than $3 \sigma$ (where $\sigma$ is the standard deviation of the residuals) may belong to an object and are therefore removed from the polynomial fit. As changes in the sky background from one column to another are very small, the solution found for one column is used as initial conditions for the next column.

This method is fast but is inconvenient in the case of strong gradients, as mentioned above. However, it can be used in the case of moderate gradients, like those caused by a globular cluster for example.

\subsubsection{Median filter}

The principle underlying the classical median filter consists, for each pixel of the image, in searching in a square of $n \times n$ pixels (with $n \geq 3$ and odd) centered on this pixel for the median value of the flux of the pixels, and then substracting this value from the flux of the central pixel. In our case, $n$ must be large enough so that the filter can encompass the largest detected objects. For this reason, a value of $n=15$ was assumed (Fig. 2).

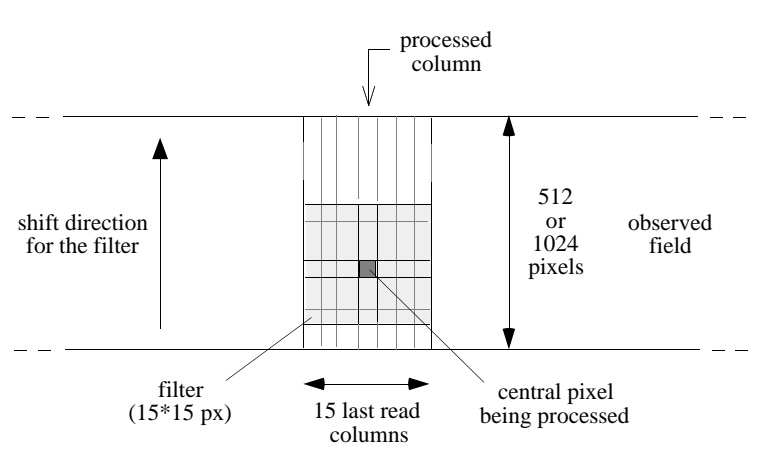

Fig. 2. Principle of the median filter

The search for the flux median value of the 225 pixels which make up the filter, when it is done for each of the pixels of the field, requires a lot of computation time and slows down the image processing considerably. That is why a slightly different method was used here. The principle of this method is first to search for the median value $M h(i)$ of the flux for the pixels along each row $i$ of the filter (i.e. horizontal median), then to search among the 15 values of the flux $M h(i)$ obtained for the median value $M$ (i.e. vertical median). $M$ is then substracted from the flux of the central pixel of the filter. This method is much faster than the classical method and results from both methods are very close. No pixel rejection is made when searching for the median value of the flux.

\subsection{Detection and identification of the objects}

After extraction of the sky background, the standard deviation $\sigma$ of the residuals of the pixels of the processed column is calculated. Pixels with residuals greater than $3 \sigma$ may belong to an object. In order to avoid as far as possible identifying some possible parasitic effects with actual objects, an object is defined by at least 2 consecutive pixels with residuals greater than $3 \sigma$. Detection threshold may be lowered down to $2.5 \sigma$ when searching for faint objects. Limits of the object are searched for in the processed column and in the following ones, then a detection window is defined surrounding the object. A security margin of 2 pixels in each direction was added between the edges of the object and the limits of the window, in order to ensure that the whole object is contained in the window. True local sidereal time (equal to the apparent right ascension when the object is on the meridian) and an approximation of the apparent declination of the centre of the window are known and compared with the apparent positions of the stars in a reference catalogue. If there is agreement to within \pm 3 pixels, the object is identified with the star giving the best agreement. If not, a temporary identification is given.

\subsection{Position and profile of the objects}

The search for the photocentre of the object is made by using a two-dimensional Gaussian flux distribution:

$$
\begin{aligned}
\phi(x, y)= & \frac{\Phi}{2 \pi \sigma_{x} \sigma_{y} \sqrt{1-\rho^{2}}} \mathrm{e}^{-\frac{1}{2\left(1-\rho^{2}\right)}\left[\left(\frac{x-\mu_{x}}{\sigma_{x}}\right)^{2}+\left(\frac{y-\mu_{y}}{\sigma_{y}}\right)^{2}\right.} \\
& \left.-2 \rho\left(\frac{x-\mu_{x}}{\sigma_{x}}\right)\left(\frac{y-\sigma_{y}}{\sigma_{y}}\right)\right]
\end{aligned}
$$

where $\Phi$ is the total flux of the object, $\left(\mu_{x}, \mu_{y}\right)$ is the centre of the Gaussian, $\sigma_{x}$ and $\sigma_{y}$ are the standard deviations in $x$ and $y$ of the Gaussian and $\rho$ is the correlation coefficient between the two dimensions of the Gaussian. Equation (1), when written for each pixel of the object, leads to a non linear system which has to be solved. That is why for each pixel $(i, j)$ a linearization is 
made:

$$
\begin{aligned}
\phi_{0}(i, j)-\phi(i, j)= & \frac{\partial \phi}{\partial \Phi} \Delta \Phi+\frac{\partial \phi}{\partial \mu_{x}} \Delta \mu_{x}+\frac{\partial \phi}{\partial \mu_{y}} \Delta \mu_{y} \\
& +\frac{\partial \phi}{\partial \sigma_{x}} \Delta \sigma_{x}+\frac{\partial \phi}{\partial \sigma_{y}} \Delta \sigma_{y}+\frac{\partial \phi}{\partial \rho} \Delta \rho
\end{aligned}
$$

where $\phi_{0}(i, j)$ is the flux received by pixel $(i, j), \phi(i, j)$ is the corresponding flux calculated by formula (1), and $\Delta \Phi, \Delta \mu_{x}, \Delta \mu_{y}, \Delta \sigma_{x}, \Delta \sigma_{y}, \Delta \rho$ are the unknowns of the system. This system is solved by least-squares and by successive approximations. This means that the barycentre of the image is first calculated and then used as initial conditions for fitting a circular Gaussian (i.e. $\sigma_{x}=\sigma_{y}$ and $\rho=0$ ). If the circular Gaussian converges, fit of a non-circular Gaussian is attempted. If the non-circular Gaussian does not converge, the assumed flux distribution is the one given by the circular Gaussian. If the circular Gaussian did not converge, the photocentre is assumed to be the barycentre of the image.

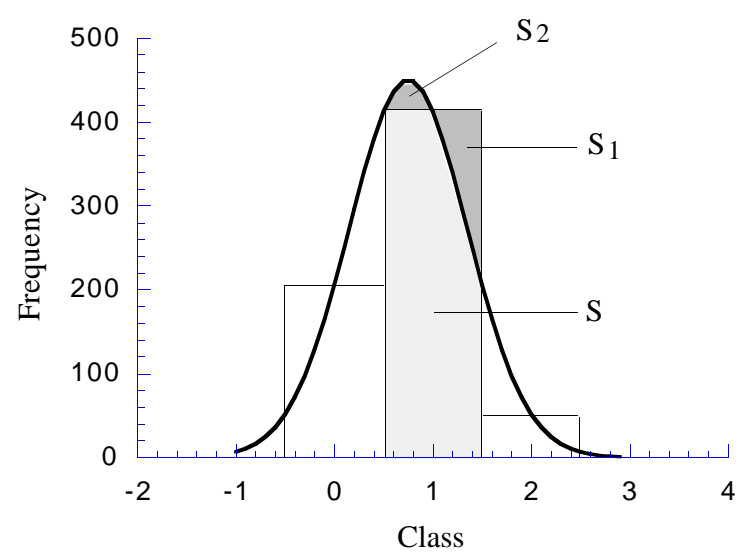

Fig. 3. Undersampling case: surfaces $S 1$ and $S 2$ are clearly different. So here, the difference between the flux received on the pixel (surfaces $S+S 1$ ) and the corresponding flux given by the Gaussian (surfaces $S+S 2$ ) is certainly significant

In most cases, the system is undersampled, that is to say, the size of the pixel (1.65 or $\left.1.51^{\prime \prime}\right)$ is too large as compared with the standard deviation of the Gaussian (about 0.7 pixel in good conditions). In these cases, the difference between the received flux on a pixel and the corresponding flux given by the Gaussian for this pixel is significant (Fig. 3). This phenomenon leads to a bias in the determination of the photocentre of the star which depends on the position of the photocentre with respect to the pixel. To solve this problem, a more exacting calculation was made, by replacing in formula $(2) \phi(i, j)$ with $\phi_{\text {cor }}(i, j)$, with: $\phi_{\text {cor }}(i, j)=\int_{i-\frac{1}{2}}^{i+\frac{1}{2}} \int_{j-\frac{1}{2}}^{j+\frac{1}{2}} \phi(x, y) \mathrm{d} x \mathrm{~d} y$ wherein $\phi(x, y)$ is given by formula (1). In order to simplify the calculation of $\phi_{\text {cor }}$, a Taylor series expansion of $\phi(x, y)$ to the $2^{\text {nd }}$ order in $x$ and $y$ was made in the vicinity of $(i, j)$, with the condition $\{-1 / 2 \leq x-i \leq 1 / 2,-1 / 2 \leq$ $y-j \leq 1 / 2\}$. In this expansion, terms depending on $\rho$ are considered to be negligible. The result is:

$\phi_{\text {cor }}(i, j)=\phi(i, j)\left\{1+\frac{r_{x}^{2}-1}{24 \sigma_{x}^{2}}+\frac{r_{y}^{2}-1}{24 \sigma_{y}^{2}}\right\}$

with:

$$
r_{x}=\frac{i-\mu_{x}}{\sigma_{x}}, r_{y}=\frac{i-\mu_{y}}{\sigma_{y}}
$$

which is equivalent to applying a correction to $\phi(i, j)$.

Another solution to the problem of undersampling, which may be combined with the preceding solution, is to defocus star images, leading to a larger FWHM (full width at half maximum) of the Gaussian, as Stone et al. (1996) did on the Flagstaff Astrometric Scanning Transit Telescope.

Dynamical range for the CCD receptor is about $7 \mathrm{mag}$. For bright objects ( $V \leq 8.5$ in most cases), some pixels of the object are saturated. These pixels are not used for fitting the Gaussian, in order to avoid as far as possible any loss in precision on the determination of the photocentre and any bias on the measurement of the flux of these objects.

On the other hand, if multiple objects have been detected (i.e. several maxima appear in the square of pixels surrounding the main image), several Gaussians are fitted simultaneously in order to enable the position and the flux of each object to be measured independently, when separation between the objects is larger than $5^{\prime \prime}$.

\subsection{Case of high declinations}

In drift scan mode, the rate of motion of electric charges in the detector is matched with the mean rate of transit of the star image in the field. The differential transit rate of the star image between the top and the bottom of the CCD detector increases with declination. In the case of large declinations (positive or negative), some limitations appear because the differential transit rate between the centre and the borders of the field is too high. Another problem is the fact that the star in front of the detector follows a curved path (due to the curvature of the parallel, which increases with declination). In the case of large declinations, both problems result in distorsion of the star's image and loss of precision in its measurement, which is greater if the star is near the borders of the field. Accordingly, stars for which the elongation of their image along the $x$ axis is greater than $6 \sigma$ (where $\sigma$ is the standard deviation of the star measurement) cannot be used for reduction and are rejected. With the $1024 \times 1024$ detector, the problem becomes significant for declinations above about 
$45^{\circ}$. At $\delta=60^{\circ}$, about $50 \%$ of the field is rejected. At Valinhos, this problem is less critical because the size of the detector is twice as small. In all cases, declinations above $\pm 77.8^{\circ}$ are out of reach because of electronic limitations.

\section{Reduction procedure}

The field to be measured is covered by coincident or overlapping strips scanned during different nights. An overall iterative reduction procedure, which is described in the following, is applied. This procedure uses the Gauss-Seidel algorithm (see Rapaport \& Le Campion 1990, for more details).

\subsection{Reduction of the strips of each night}

For each strip, the following equations are written down for each reference star:

$$
\left\{\begin{array}{l}
\alpha_{\mathrm{REF}}=\alpha_{0}+a_{1} x+a_{2}\left(y-y_{0}\right) \\
\delta_{\mathrm{REF}}=\delta_{0}+b_{1}\left(y-y_{0}\right)+b_{2} x[+c \Phi] \\
V_{\mathrm{REF}}=V_{0}+[d x]-2.5 \log \Phi
\end{array}\right.
$$

where $\alpha_{0}$ is the local sidereal time corresponding to the beginning of the strip, $x$ and $y$ are the measured coordinates (in pixels) of the star, $\Phi$ is its flux measured in encoder step units, $y_{0}$ is the approximate centre of the strip and $\delta_{0}$ is the corresponding declination of the field. In the case of saturated bright stars, attempts were made to correct the possible bias caused by faulty charge transfer in declination, by adding the optional term $[+c \Phi]$ in the declination equation; however, this point is still under consideration. In the magnitude equation, the optional $x$ dependent term (i.e. a function of time) may be added if quality of the observing conditions changed during the observation of the field.

In Eqs. (3), coordinates $\alpha_{\mathrm{REF}}$ and $\delta_{\mathrm{REF}}$ and magnitude $V_{\mathrm{REF}}$ of the reference stars are taken from one or several reference catalogues (Sect. 4.5). These coordinates are reduced at the epoch of observation by using the associated proper motions and then converted to apparent coordinates.

The system is solved by least-squares, in a procedure in which the reference stars are rejected if their residuals are in excess of $3 \sigma$, where $\sigma$ is the standard deviation of the residuals for all the reference stars. New positions and magnitudes are then calculated for all the stars present in the field by using the resulting instrumental constants $\alpha_{0}, a_{1}, a_{2}, \delta_{0}, b_{1}, b_{2}, c, V_{0}, d$ and Eqs. (3).

\subsection{Global reduction}

When all the strips have been separately reduced, mean positions and magnitudes of all the stars present in all the corresponding fields are calculated, and a preliminary mean catalogue is obtained. This catalogue is re-introduced as a reference catalogue in the reduction procedure described in Sect. 4.1, and the entire procedure is reiterated several times (i.e. the strips are separately reduced again, then a new mean position and magnitude is calculated for all the stars, giving a new mean catalogue, etc...) for obtaining the global solution (Eichhorn 1960; Benevides-Soares \& Teixeira 1992; Teixeira et al. 1992). In most cases, convergence is reached after 5 or 6 iterations.

From the first iteration, oscillations emerging from measurement noise can be seen when comparing the catalogues resulting from the reduction of each strip with the mean catalogue (Fig. 4a). These oscillations are mostly caused by long-period atmospheric fluctuations (primarily between 4 and 30 minutes). They represent a signal which is not taken into account in the reduction procedure described above. Fitting a curve by the B-spline method on the residuals of each night is suggested for attenuating the oscillations. Effects of atmospheric fluctuations on the observations have been investigated by Høg (1968), Lazorenko (1992) and Benevides-Soares et al. (1993).

\subsection{B-spline method}

With the B-spline method, nodes are fixed with a given step in $x$ for each strip, ordinates are adjusted by least-squares on the residuals and a B-spline is fitted onto the nodes. This curve satisfies the cubic spline conditions for each node $\left(X_{i}, 1 \leq i \leq n\right)$. The nodes are spaced in such a way that there are at least 20 stars between 2 nodes. In all cases, the space between 2 nodes is at least equal to the integration period divided by 2.5 , i.e. $46 \mathrm{~s}$ in Bordeaux and $23 \mathrm{~s}$ in Valinhos. Indeed, the minimal period of the atmospheric fluctuations which is primarily present in the residuals is equal to the integration period, and simulations have shown that a signal of period $P$ is correctly modelled by the B-spline with a interval between 2 nodes equal to $P / 2.5$. There is one B-spline for every strip which is introduced from the second iteration in Eqs. (3) of the preliminary reductions of the strips, as follows:

$$
\left\{\begin{array}{l}
\alpha_{\mathrm{REF}}=\alpha_{0}+a_{1} x+a_{2}\left(y-y_{0}\right)+B \operatorname{spline}_{\alpha}(x) \\
\delta_{\mathrm{REF}}=\delta_{0}+b_{1}\left(y-y_{0}\right)+b_{2} x[+c \Phi]+B \operatorname{spline}_{\delta}(x) \\
V_{\mathrm{REF}}=V_{0}+[d x]-2.5 \log \Phi+B \operatorname{spline}_{V}(x)
\end{array}\right.
$$

It is re-calculated at every iteration by using all the stars within a $3 \sigma$-rejection threshold. An example of a B-spline fitted to the residuals of a strip with respect to the mean catalogue is shown in Fig. 4b. Then, the B-spline is substracted from the observed positions of all the stars of the strip. Residuals of the preliminary catalogue of the 


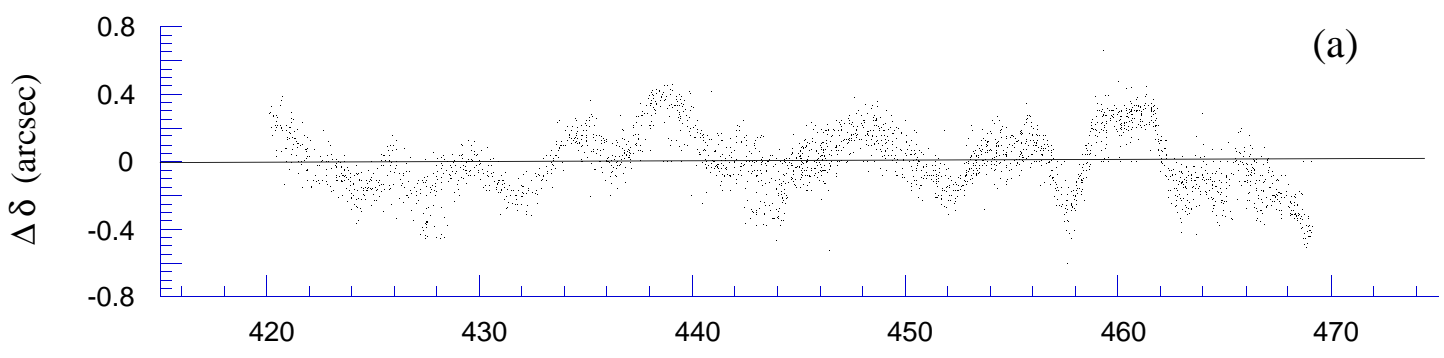

Right ascension (minutes)
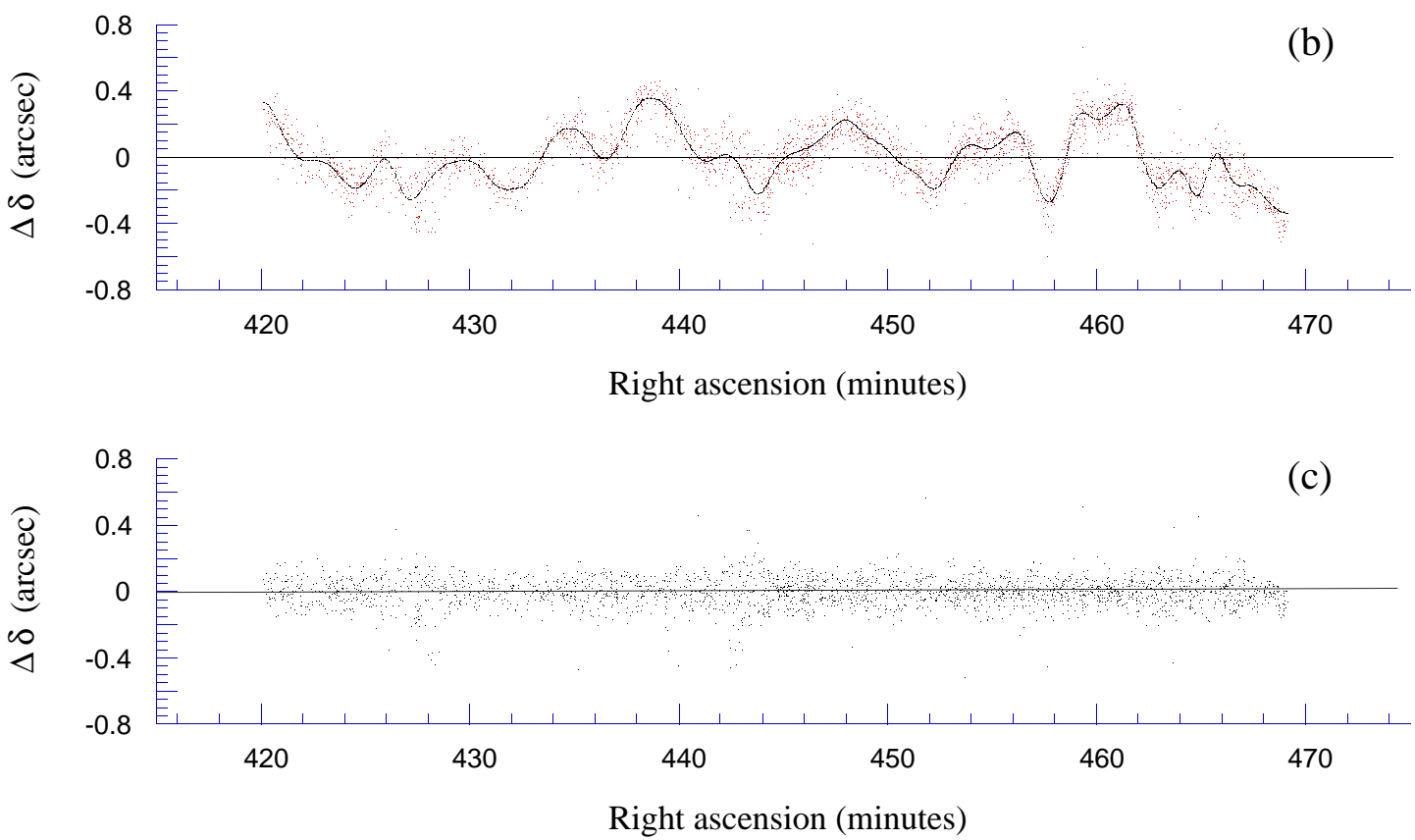

Fig. 4. a) Example of residuals in declination between the catalogue obtained with a preliminary reduction of a strip and the mean catalogue: case of a field around the N2384 open cluster. This example intentionally corresponds to severe observing conditions in Bordeaux, at declinations where atmospheric turbulence is very significant (zenith distance: $66^{\circ}$ ). Only residuals for stars up to $V=15$ have been plotted. Standard deviation of these residuals: $0.180^{\prime \prime}$. b) Fitting of a B-spline on the residuals of previous figure. c) Same as a) after substraction of the B-spline. Standard deviation of the residuals is now $0.076^{\prime \prime}$

strip with respect to the mean catalogue after substraction of the B-spline are shown in Fig. 4c. It can be seen that the standard deviation of the residuals has decreased from $0.180^{\prime \prime}$ to $0.076^{\prime \prime}$.

\subsection{Final B-spline}

At the end of the reduction, the final catalogue obtained is expressed in a local reference system and approximately retains the orientation of the primary reference catalogue. The residuals (final catalogue - primary catalogue) for the reference stars simultaneously present in both catalogues may exhibit oscillations which are significant with respect to the scattering of the residuals, when residual atmospheric turbulence exceeds the internal precision of the primary catalogue (with the Tycho catalogue, case of large zenith distances or when the number of nights is too small). Fitting of a final B-spline on these residuals is proposed in those cases for tying more rigorously the final catalogue to the frame of the primary reference catalogue. To distinguish this final B-spline from the B-splines introduced in Sect. 4.3 which are specific to each strip, the latter will be referred to as "individual B-splines" in the following. The complete reduction procedure is summarized in Fig. 5.

\subsection{Reference catalogue}

For selecting the catalogue of reference stars, two parameters have to be considered:

- The declination field of the cameras is limited to $28^{\prime}$ (Bordeaux) and 13' (Valinhos), respectively. Since the length of the scanned strips is typically contained between 30 minutes and 1 hour, the corresponding fields are generally contained between 3 and 7 square degrees. Therefore a 


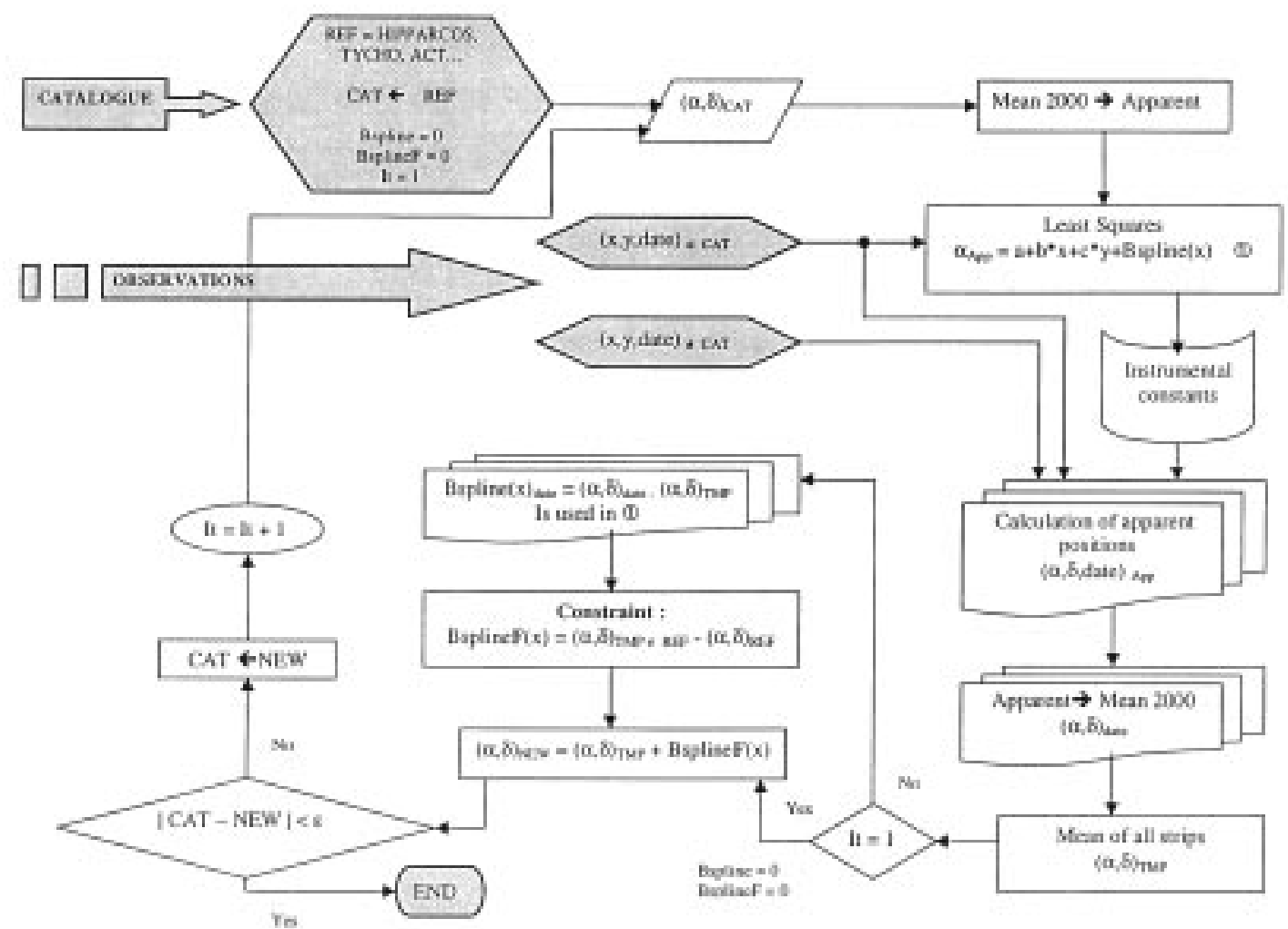

Fig. 5. Summary of the complete reduction procedure

reference catalogue with at least 3 stars per square degree is needed.

- The saturation effect appears for stars brighter than about $8.5 \mathrm{mag}$ and results in a varying systematic error in declination. The Hipparcos catalogue has just the required star density but in most cases a great number of stars have to be rejected because of the saturation effect.

Our reference catalogue finally comprises the Hipparcos catalogue and the ACT catalogue for those Tycho stars which are not present in the Hipparcos catalogue. The ACT catalogue contains the Tycho positions, associated with proper motions obtained by combining the J1991.25 Tycho positions with the AC 2000 catalogue (Urban et al. 1998b). Both Hipparcos and Tycho catalogues refer to the International Celestial Reference System (ICRS). Remember that the internal precision of the Hipparcos catalogue is about 1 mas in both coordinates for positions and $1 \mathrm{mas} / \mathrm{yr}$ for proper motions (ESA 1997a). The internal median standard error of the J1991.25 Tycho positions is about 25 mas, with a median magnitude of about 10.5 (ESA 1997b). However it was found that standard errors in the Tycho catalogue are underestimated for stars fainter than $V \simeq 9.5 \mathrm{mag}$, typically for $V=10.5 \mathrm{mag}$ a factor of 1.5 should be applied (ESA 1997c). A median error of about $35-40$ mas at J1991.25 therefore seems to be more realistic. Uncertainty in proper motions is also important for estimating the true error of the reference catalogue positions at the epoch of the observations. Accuracy of ACT proper motions is expected to be about $3 \mathrm{mas} / \mathrm{yr}$, much better than that of the original Tycho proper motions for magnitudes greater than 9.0 . The value of $3 \mathrm{mas} / \mathrm{yr}$ seems to be realistic within the magnitude range $[5-10.5]$, as recently shown by a comparison with Hipparcos proper motions (Froeschlé 1998).

\section{Typical results}

\subsection{Limiting magnitude}

The limiting magnitude is influenced by a lot of factors like integration time, brightness of the sky background, sky transparency, optics of the instrument, filters used, quality of focusing and electronic noise. An example of a limiting magnitude obtained in Bordeaux under good conditions (near the zenith and with good focusing) is given in Fig. 6. This example shows the number of stars which have been seen at least twice per 0.1 mag interval, in order to remove parasitic effects. The increase in the number of stars versus magnitude corresponds to the star distribution in the sky, until about $V=16$. Thereafter, a very sharp drop occurs in the number of detected stars, and the detection threshold is about $V=16.6$. Similar results are obtained with the Valinhos CCD meridian circle. 


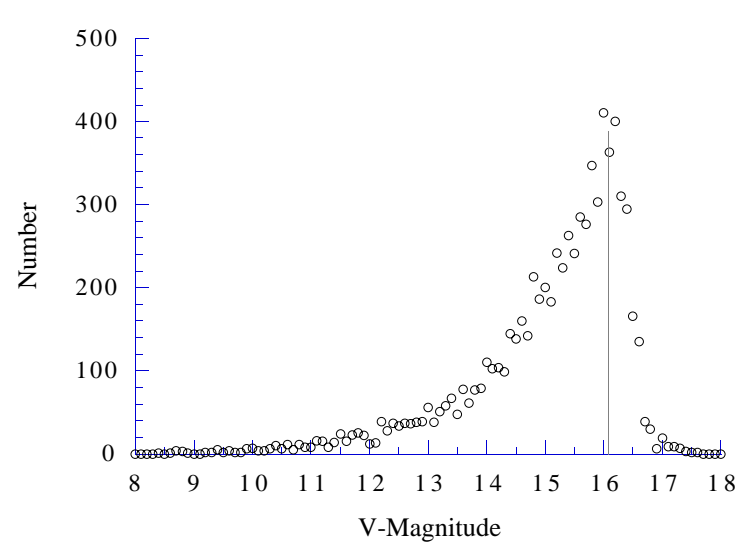

Fig. 6. Number of stars, per $0.1 \mathrm{mag}$ interval, in the field around the M 92 globular cluster, seen at least twice in a simultaneous reduction of 8 strips. Location: Bordeaux. Zenith distance: about $2^{\circ}$

\subsection{Detection of stars: Comparison with GSC}

A comparison between a field around M 35 observed in Bordeaux and the corresponding area of the Guide Star Catalogue has shown that about $99 \%$ of the GSC stars were found in the field observed in Bordeaux. Identification of the stars was made when discrepancy between meridian and GSC positions was smaller than $6^{\prime \prime}$ in each coordinate and when discrepancy in magnitude was smaller than $2 \mathrm{mag}$. The $1 \%$ of stars not found may be due to large amplitude variable stars, stars with large proper motions (the mean epoch difference between the GSC and meridian observations is about 15 years), or also badly known double stars.

On the other hand, the converse comparison was also made. The proportion of stars present in the meridian reductions of the field around $M 35$ which were also found in the GSC was calculated and plotted versus the meridian $V$-magnitude (Fig. 7). It can be seen in this figure that for this field the GSC is not complete after $V=12$, with a limiting magnitude of about $V=16$ (this fact is explained in Lasker et al. 1988). Accordingly, the number of stars detected by the meridian in this area is about 4 times greater than the number of GSC stars.

\subsection{Internal positional error}

After preliminary reduction of a typical field at a moderate zenith distance (field around M $35, z=21^{\circ}$ ), the standard error for a single observation of a star (regardless of the reference catalogue's accuracy) is about $\pm 0.06^{\prime \prime}$ for $9 \leq$ $V \leq 14$, and $\pm 0.17^{\prime \prime}$ for $V=15.5$ in both coordinates, as shown in Fig. 8. When individual B-splines are used, this standard error becomes about $\pm 0.04^{\prime \prime}$ for $9 \leq V \leq 14$, and $\pm 0.15^{\prime \prime}$ for $V=15.5$, as shown in Fig. 9. In both cases, these standard errors were obtained by reducing 18 overlapping strips (total size of the field: 1 h15 in R.A.,

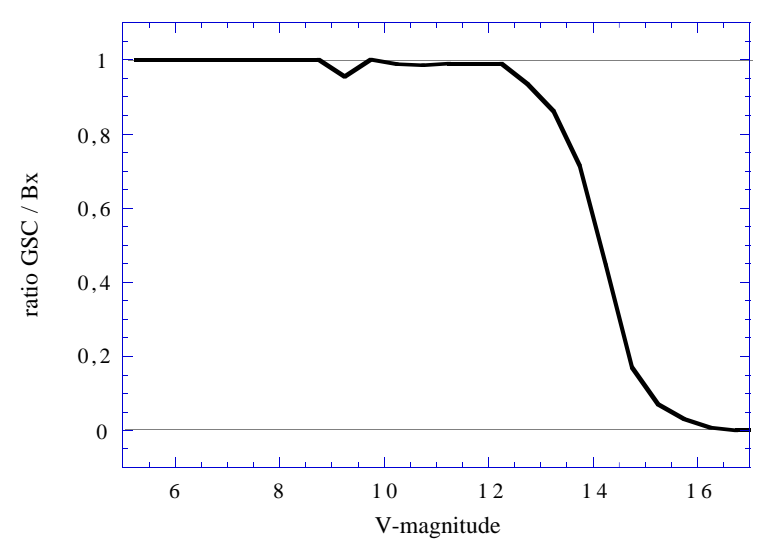

Fig. 7. Ratio of the number of stars in GSC versus the number of meridian stars per $V$-magnitude interval in a field of $1 \mathrm{~h} \times 0.5^{\circ}$ around M 35 as observed in Bordeaux

$1^{\circ}$ in Decl.) and calculating for each star the standard deviation of its residuals with respect to its mean position. No selection of the strips used for this calculation was made, so that the selected example is a typical one. When selecting the best strips, the standard error of a single observation decreases down to about $\pm 0.03^{\prime \prime}$ for $9 \leq V \leq$ 14 with individual B-splines.

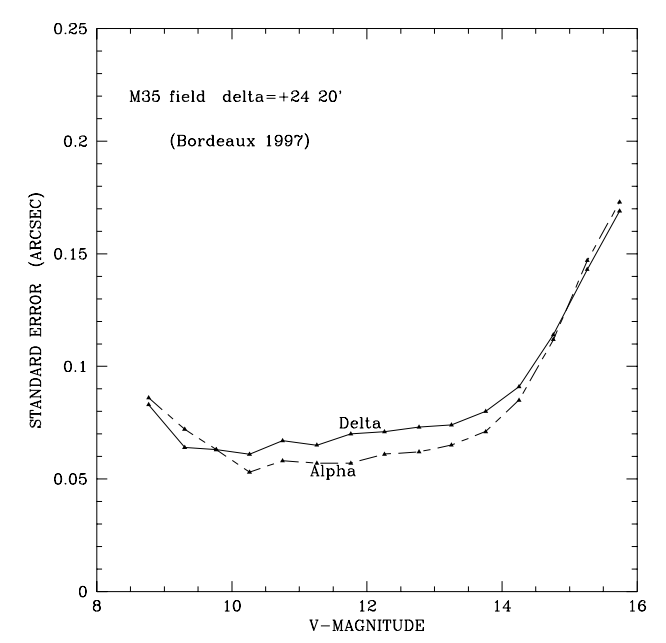

Fig. 8. Standard error in right ascension $(\times \cos \delta)$ and declination of a single observation of a star as a function of magnitude for a field around M 35 as observed in Bordeaux

On both figures, it can be seen that standard error rapidly increases for faint objects beyond $V=14$, because the signal to noise ratio is too low. For bright objects $(V \leq 9)$, precision is worse because of signal saturation. Another fact lies in the improvement in precision induced by the use of individual B-splines, which is clearly apparent after comparison of both figures. Moreover, relevance of using B-splines increases with the zenith distance of the 


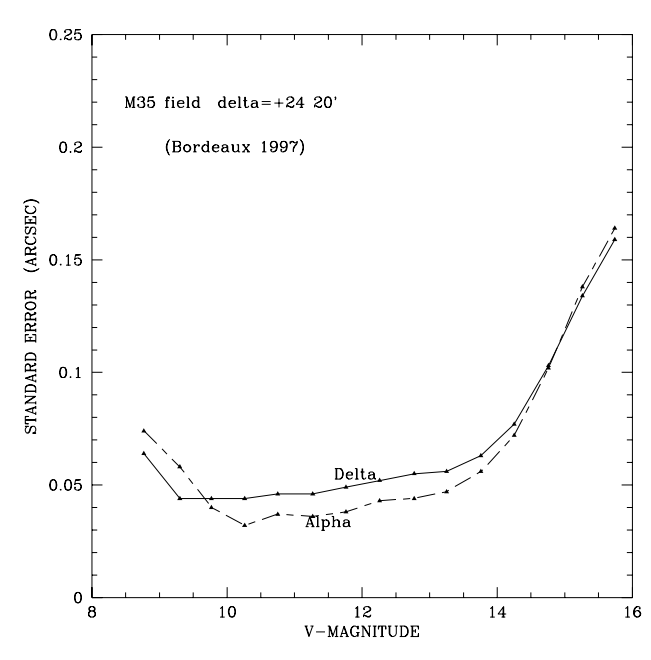

Fig. 9. Same as Fig. 8, but after using individual B-splines in the reduction procedure

observation, as atmospheric turbulence also increases. In a very critical case (example of cluster N2384, $\delta=-21^{\circ}$, $z=66^{\circ}$ in Bordeaux), the precision of a single position at $V=12$ falls from $0.12^{\prime \prime}$ in R.A. (i.e. $\alpha \cos \delta$ ) and $0.18^{\prime \prime}$ in Decl. to about $0.06^{\prime \prime}$ and $0.08^{\prime \prime}$, respectively, which confirms the results shown in Fig. 4, in Sect. 4.3.

Also, it can be seen that precision is slightly better in right ascension than in declination. This is more apparent in the case of N2384, where the zenith distance is large, and it may be caused by the effects of atmospheric turbulence. The fact that the mean error in declination is more sensitive to the zenith distance than error in right ascension has already be mentioned in Morrison (1985-94).

\subsection{Comparisons with the primary reference catalogue}

Differences between coordinates of the reference stars obtained after reduction and coordinates of these stars given in the primary reference catalogue (see Sect. 4.5) were calculated for a set of typical fields observed in Bordeaux and in Valinhos. Standard deviations of these differences were calculated for all the stars seen at least 4 times, and are given in Table 2. They are due on the one hand, to the measurement error of the star image, (as described in Sect. 5.3) and on the other hand, to the accuracy of the reference catalogue (Sect. 4.5).

The strips were reduced by using individual B-splines. In the case of N2384, for which atmospheric turbulence effects are important because of its large zenith distance, a final B-spline, as described in Sect. 4.4, was added. The four examples of fields observed in Bordeaux cover a large zenith distance interval. It can be seen that standard deviation of residuals in declination tends to increase with zenith distance, which may be a direct effect of atmospheric turbulence. On the other hand, there is no apparent correlation between zenith distance and standard deviations of residuals in right ascension and magnitude.

As for photometry, it can be seen in Table 2 that mean standard deviations of the differences (meridian - reference catalogue) range from $0.10 \mathrm{mag}$ to about $0.18 \mathrm{mag}$, and in most cases a mean value of 0.15 mag can be considered. However, internal photometric precision is better, about $0.05 \mathrm{mag}$ for $9 \leq V \leq 14$. More details about photometry are given in Sect. 5.8 .

\subsection{External positional error}

In the two following examples, selected at two very different zenith distances, we only used the ACT stars not included in the Hipparcos catalogue as reference. The Hipparcos stars are therefore considered as target stars and are used to record the accuracy of the reduction of the considered fields. In both examples, differences between the observed positions of the Hipparcos stars and their positions taken in the Hipparcos catalogue were calculated.

\subsubsection{Bordeaux CCD observations of M 35 cluster}

The first example is the field surrounding the M 35 cluster. In this example, only stars fainter than $V=8$ and seen at least 3 times have been retained. The reduction procedure has been made with individual B-splines and without the final B-spline. Standard deviation of residuals is $0.038^{\prime \prime}$ in R.A. and $0.045^{\prime \prime}$ in Decl. The only out-of-bounds object in declination is a double star, according to the Hipparcos catalogue (HIP 27135).

\subsubsection{Bordeaux CCD observations of N2384 cluster}

The second example is the field around the N2384 open cluster $\left(\delta=-21^{\circ}\right)$. The residuals for the Hipparcos stars are shown in Fig. 11. Each star was observed 4 to 8 times. The two anomalous out-of-bounds objects correspond to two close double stars according to the Hipparcos catalogue. Standard deviation on the differences for the 21 Hipparcos stars is $0.027^{\prime \prime}$ in R.A. and $0.049^{\prime \prime}$ in Decl. These results, obtained at a zenith distance of $66^{\circ}$ and during about one hour, are close to those obtained for M 35. They show that the effects of substantial atmospheric turbulence are practically removed by the B-spline reduction procedure and confirm the results shown in Sects. 4.3 and 5.3 . 
Table 2. Standard deviations of the differences (meridian - reference catalogue) for all the reference stars seen at least 4 times. $z$ is the mean zenith distance of the field

\begin{tabular}{|c|c|c|c|c|c|c|c|c|c|c|c|c|c|}
\hline \multirow[t]{2}{*}{$\begin{array}{l}\text { Name of } \\
\text { the field }\end{array}$} & \multirow[t]{2}{*}{ Location } & \multirow[t]{2}{*}{$\begin{array}{l}\text { Number } \\
\text { of nights }\end{array}$} & \multicolumn{5}{|c|}{ Limits of the field } & \multicolumn{3}{|c|}{ Number of stars } & \multicolumn{3}{|c|}{$\begin{array}{c}\text { Standard deviation } \\
\text { of residuals }\end{array}$} \\
\hline & & & $\begin{array}{l}\alpha_{\min } \\
\mathrm{h} \min \end{array}$ & $\begin{array}{l}\alpha_{\max } \\
\mathrm{h} \min \end{array}$ & $\delta_{\substack{0^{\prime} \\
\text { min }}}$ & $\delta_{\substack{\max ^{\prime}\\
}}$ & $\begin{array}{l}z \\
\circ\end{array}$ & $N_{\alpha}$ & $N_{\delta}$ & $N_{V}$ & $\begin{array}{c}\sigma_{\alpha} \\
\prime \prime\end{array}$ & $\begin{array}{c}\sigma_{\delta} \\
\prime \prime\end{array}$ & $\begin{array}{c}\sigma_{V} \\
\operatorname{mag}\end{array}$ \\
\hline M 35 & $\mathrm{Bx}$ & 18 & 0503 & 0649 & +2348 & +2446 & 21 & 318 & 314 & 321 & 0.060 & 0.053 & 0.166 \\
\hline $3 \mathrm{C} 273$ & $\mathrm{Bx}$ & 8 & 1144 & 1259 & +0149 & +0218 & 43 & 44 & 44 & 38 & 0.065 & 0.058 & 0.125 \\
\hline Pluto & $\mathrm{Bx}$ & 16 & 1530 & 1711 & -0851 & -0759 & 53 & 124 & 128 & 120 & 0.059 & 0.073 & 0.178 \\
\hline N2384 & $\mathrm{Bx}$ & 7 & 0654 & 0754 & -2118 & -2041 & 66 & 321 & 329 & 330 & 0.059 & 0.082 & 0.171 \\
\hline $3 \mathrm{C} 273$ & Val. & 40 & 1200 & 1300 & +0155 & +0210 & 21 & 36 & 36 & 36 & 0.083 & 0.086 & 0.109 \\
\hline Q2005 & Val. & 33 & 1930 & 2030 & -4900 & -4845 & 26 & 74 & 74 & 74 & 0.084 & 0.053 & 0.144 \\
\hline
\end{tabular}

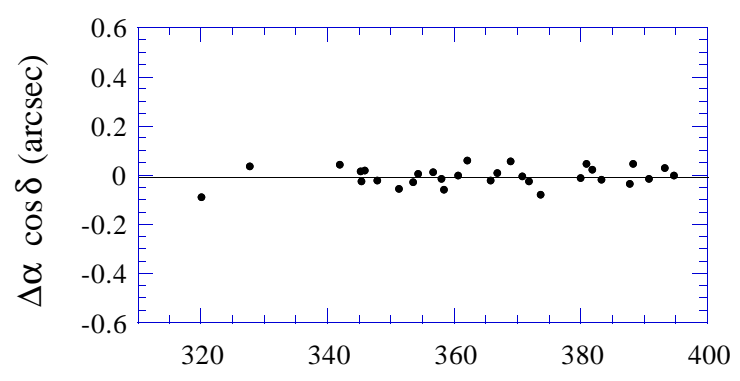

Right ascension in minutes

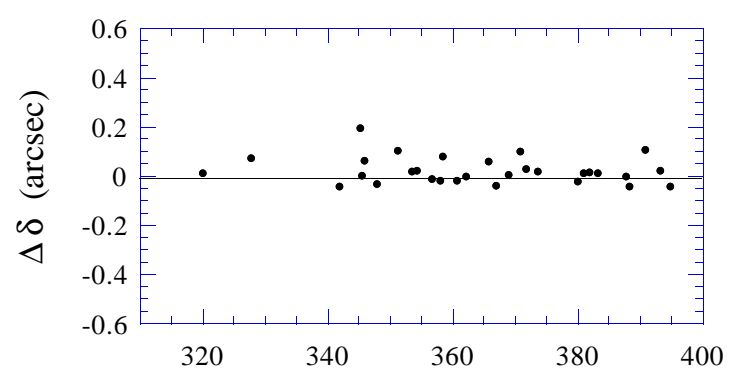

Right ascension in minutes

Fig. 10. Differences (Bordeaux-Hipparcos) at the epoch 1997.0 for 30 Hipparcos stars considered as target stars around M 35 $\left(\delta=+24^{\circ}, z=21^{\circ}\right)$

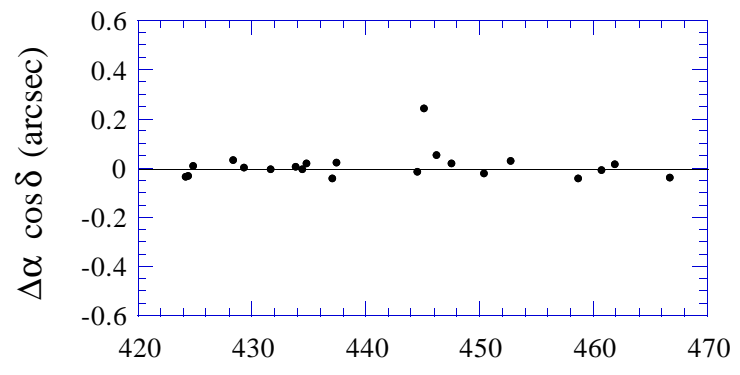

Right ascension in minutes

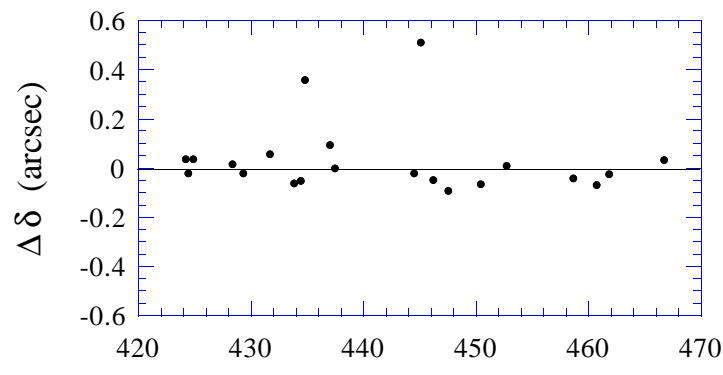

Right ascension in minutes

Fig. 11. Differences (Bordeaux-Hipparcos) at the epoch 1997.1 for 21 Hipparcos stars considered as target stars around N2384 $\left(\delta=-21^{\circ}, z=66^{\circ}\right)$

\subsection{Bordeaux and Valinhos CCD observations of Pluto}

Figure 12 shows the differences between Bordeaux and Valinhos observations of Pluto obtained in 1997 and the new JPL ephemerides DE405 kindly provided by E.M. Standish. The declination of Pluto was about $-8^{\circ}$ (i.e. $z=53^{\circ}$ in Bordeaux and $15^{\circ}$ in Valinhos) with a mean $V$ magnitude of 14.2 . Due to the slow motion of Pluto on its orbit, there were overlappings between the different strips, which were reduced with individual B-splines. The standard error on the differences (Bordeaux and Valinhos) is here $0.076^{\prime \prime}$ in R.A. and $0.089^{\prime \prime}$ in Decl., which is consistent with Figs. 8 and 9. Observations and ephemerides show very good agreement on this figure, and no system- atic effect between Bordeaux and Valinhos residuals can be seen.

\subsection{Bordeaux CCD observations of the asteroid (32) \\ Pomona}

Figure 13 shows residuals of observations of (32) Pomona made in Bordeaux $\left(V=12, \delta=+16^{\circ}, z=29^{\circ}\right)$. The orbital data used for the calculation of these residuals were adjusted by using all the available observations of (32) Pomona (1864-1997). There were a few overlappings between the strips, which improved the accuracy of the reduction and enabled us to use the individual Bsplines method. There is very good agreement between the 

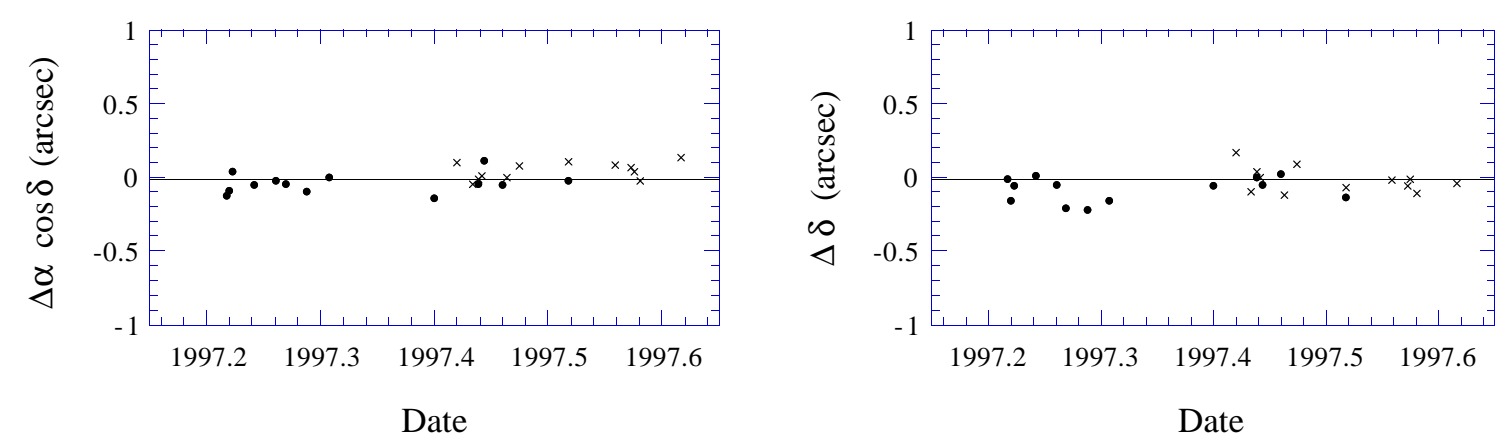

Fig. 12. Differences (Bordeaux and Valinhos)-DE405 for Pluto at the epoch 1997.5 (solid circles for Bordeaux, crosses for Valinhos)
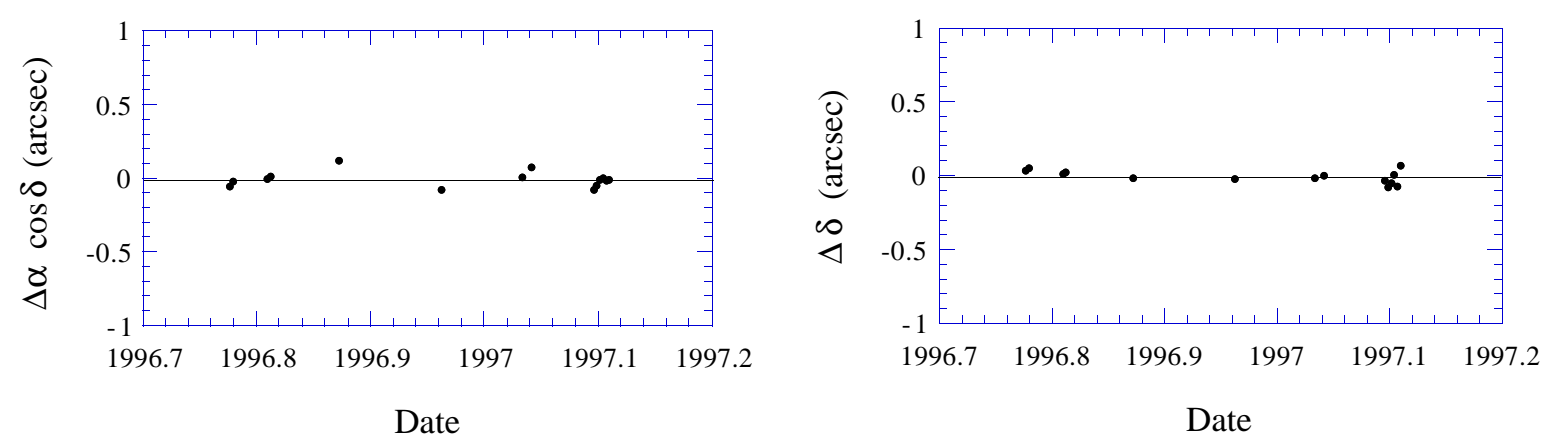

Fig. 13. Residuals of Bordeaux CCD meridian observations of the asteroid (32) Pomona, using orbital data corrected with all the available observations of Pomona

observed and calculated positions of Pomona, which indicates, as in the Pluto example, that the observed positions are free of any apparent systematic bias. Standard deviations of the residuals are very small: $0.053^{\prime \prime}$ in R.A. and $0.043^{\prime \prime}$ in Decl. These values are in agreement with those plotted in Fig. 9 (Sect. 5.3).

\subsection{Photometry}

The scanning micrometer was conceived primarily as an astrometrical device. Photometry is a by-product, useful for identification purposes. However, prior accumulated experience indicates that photometric results exhibit a high quality level, so that they deserve to be considered for their own sake.

In the reduction procedure described in Sect. 4, stellar magnitudes are entered as a third unknown parameter. The object fluxes - estimated either by summing up the pixel values inside the image rectangle or by integrating the volume under the bi-variate Gaussian fitting surface - are initially converted into a measure of magnitude by the standard formula

$V=V_{0}-2.5 \log \Phi$

where $\Phi$ is the flux and $V_{0}$ is a convenient constant.

The reference stars' magnitudes are plugged into the left handside of the above equation and the parameter $V_{0}$ is estimated by least-squares. In the case of strips of duration longer than, say, 40 minutes, a linear time dependent term must be added to the right hand side. A spline model can also be very useful in the present context.

Internal accuracy of the magnitude results falls within the range from about $0.05 \mathrm{mag}$ - for objects with $V \sim$ 12 or 13 - to $0.1 \mathrm{mag}$, near the faint magnitude limit (Fig. 14). These estimates result from the residuals with respect to the average value, for those objects that do not display obvious variability.

Significance of the photometric results is of course limited by the fact that they relate to a single large spectral bandpass, so that comparison with standard photometric systems is not straightforward. However, photometric data are important for detecting long-term variability, i.e., magnitude changes within intervals from a few days to a few months or years.

Figure 15 displays the raw data collected at Valinhos, concerning photometric variability of the BL Lac object PKS 2005-489 during 1997. The light curve is rather complex, but the observed points lie essentially within the error bars from any reasonably fitted curve. 


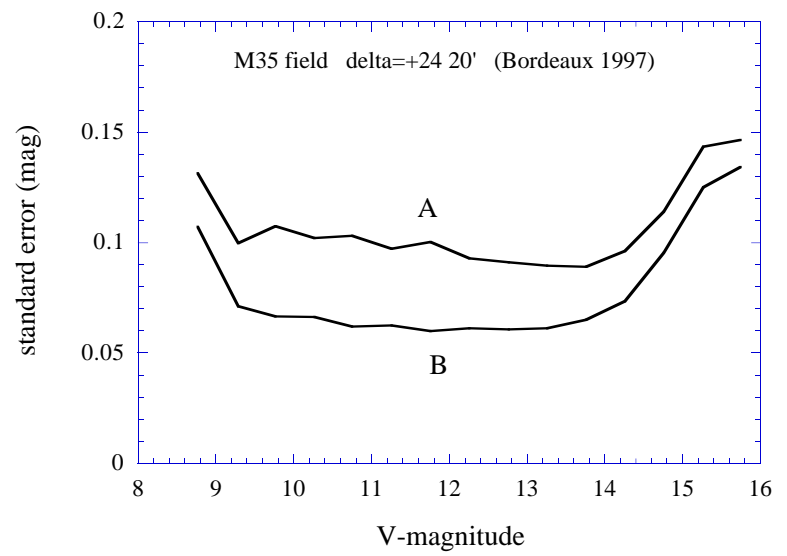

Fig. 14. Standard error in magnitude of a single observation of a star as a function of magnitude for a field around M 35 observed in Bordeaux. $A=$ reduction without B-splines, $B=$ reduction with B-splines

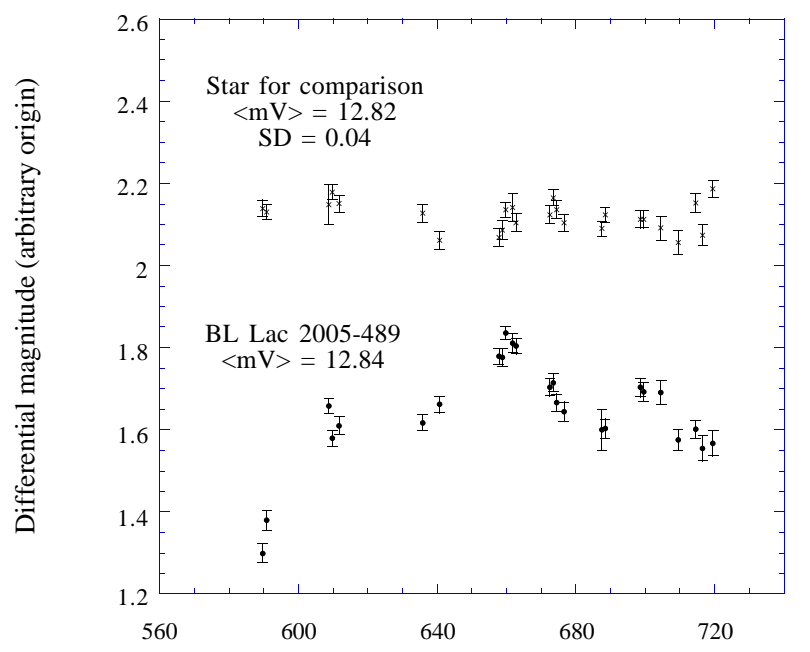

Julian Date - 2450000

Fig. 15. Photometric variability of the BL Lac object PKS 2005-489 obtained in Valinhos

\section{Ongoing programmes and projects}

\subsection{In Bordeaux}

\subsubsection{Méridien 2000}

The CCD meridian circle of Bordeaux Observatory is currently being used since January 1997 for the realization of a major scientific programme called "Méridien 2000". The object of this programme is systematic observation of the Bordeaux zone of the Astrographic Catalogue $\left(11^{\circ} \leq \delta \leq\right.$ $18^{\circ}$ ) for 3 years so as to build a catalogue of positions and proper motions for stars up to $V$-magnitude 15. Proper motions will be obtained by combining the old Carte du Ciel plates of this zone (which are to be measured with the MAMA automatic measuring machine at Paris), and also by using the AC 2000 catalogue (Uban et al. 1998a) or by re-reducing the Bordeaux zone of the Astrographic Catalogue. This programme will be presented in more detail in a future paper.

\subsubsection{Other programmes}

The Bordeaux CCD meridian circle is also used for the observation of solar system objects (outer planets, natural satellites and asteroids). Most of these programmes are jointly carried out with Valinhos.

The outer planets Uranus, Neptune (since 1997) and Pluto (since 1995) are observed for improving their ephemerides. Regular observations of Pluto are especially important because all the observations of Pluto since its discovery (1930) cover only roughly the quarter of its orbital period. Accordingly, Pluto's orbital data are rather poorly known and its ephemerides are subject to faster deterioration with elapsed time as those for other planets.

Some Saturn satellites are observed for improving their theories, which enable the centre of mass of the planet to be determined with high accuracy. The satellites can be observed even if Saturn is present on the strip by using the median filter (see Sect. 3.1.2) for extraction of the sky background.

Selected asteroids are also observed for determining the large asteroid masses, which are currently rather badly known (Viateau \& Rapaport 1998). Recent accurate observations such as the meridian observations are very useful for improving quality of the orbital parameters of the perturbed asteroid, whilst ancient observations of this asteroid are used to see the effect of gravitational perturbations induced by a large asteroid on its orbit. Observations of asteroids have also been made as "last-minute" observations in order to improve prediction of star occultations. Lastly, some known asteroids (and comets) are present in the strips observed for other topics, and are systematically searched for in the reductions of all the fields as observed in Bordeaux and Valinhos.

Among the other programmes running in Bordeaux, regions of the sky near the anticentre of the Galaxy are observed in order to determine accurate positions and proper motions for this zone, which corresponds to a small part of the Méridien 2000 zone (Odenkirchen et al. 1998). This region of the Galaxy is characterized by low absorption, high star density, and the proportion of young stars is rather high. Proper motions obtained may be used for kinematical and population studies of this zone. On the other hand, selected extragalactic sources are observed for studying their photometrical variability. Observations of fields around M 81 are also made for photometric studies. Lastly, some radio stars are also observed in order to tie the optical and the radio reference frames. 
Other programmes, such as the search for variable stars, the realization of astrometric catalogues of clusters, or the observation of a comet for the determination of nongravitational effects on its orbit are in discussion, and all proposals are welcome.

\subsection{In Valinhos}

\subsubsection{Optical positions of extragalactic sources}

This program aims to establish the link with the highest accuracy possible between the extragalactic reference frame and star positions, in the magnitude range $V=12$ to 16 . The Hipparcos catalogue contains only one extragalactic object (the quasar 3C 273), so that connection with the ICRF (International Celestial Reference Frame) was made almost exclusively by means of radio stars. The magnitude interval useful for the CCD meridian instrument extends up to $V \sim 16$, which includes about 50 ICRF sources in the appropriate declination interval. Although not all of them will be observable, due to inaccuracy or variability in the published optical magnitudes, we expect to regularly observe about 20 sources, for at least five years. First epoch data will be obtained from various sources, including the Astrographic Catalog and the DSS (Digitized Sky Survey). In addition to the direct observation of extragalactic objects, a number of fields around faint quasars were included in the program, in collaboration with the Observatorio Nacional (Rio de Janeiro, Brazil), with the purpose of establishing a secondary catalogue to be used as a reference frame for deep CCD imaging in larger telescopes. As supplementary information, a list of 600 radio stars from the Wendker catalogue (Wendker 1995) was added to the program. Observations of extragalactic sources are directed towards detecting their photometrical variability.

\subsubsection{Solar system objects}

This program relates to the positions of Uranus, Neptune and Pluto, as well as a few asteroids and satellites of the major planets. This project is conducted in collaboration with Bordeaux Observatory (see Sect. 6.1.2 above).

\subsubsection{Low extinction windows in the galactic bulge}

A number of "windows" of relatively low inter-stellar absorption have been identified in the general direction of the galactic centre, which can be associated with the galactic bulge. The first and best known of these areas is the Baade window. The program includes 12 of such windows, with the aim of obtaining an accurate description of their kinematical properties, detecting and classifying stellar variability, with an eye to the possibility of observing microlensing events due to gravitational effects from ultra-compact objects.

\subsubsection{Kinematics of open clusters and young stars}

The purpose of this project is to establish the possible connection between star formation and the trajectories of molecular clouds across the galactic disk (Lépine \& Duvert 1994). Almost a hundred clusters are candidates for this research, and ten of these are currently being observed. In the course of this work, a fairly detailed description of the cluster physical properties should be obtained. Included also in the program is a list of $35 \mathrm{~T}$ Tauri stars.

\section{Conclusion}

The CCD meridian circles of Bordeaux and Valinhos observatories have been described. Both instruments, one in each hemisphere, are complementary and can cover nearly the whole celestial sphere. These instruments are now fully operational and are involved in many programmes for several years. In particular, since their limiting visual magnitude is higher than 16 , they are used for extending the Hipparcos-Tycho reference frame.

The internal precision of the observations is about $0.04^{\prime \prime}$ in both coordinates for $9 \leq V \leq 14$. In the same magnitude range, magnitudes can also be obtained with an internal precision of about 0.05 mag. Solutions for obtaining information about the $(B-V)$ color index from the analysis of the star image are currently being tested. The knowledge of the color index of the stars, which gives information about their temperature, would also help to solve the problem of chromatic refraction, mentioned in Sect. 2.

The availability of the Hipparcos and Tycho catalogues, as also the ACT catalogue, with improved proper motions, has already provided reductions of meridian data with a high level of accuracy. In future, both instruments will certainly receive enhancements, such as smaller pixel size, thinned CCD detectors, new lenses and filters, which would result in better precision and a fainter limiting magnitude.

The Bordeaux and Valinhos CCD meridian circles work in drift scan mode. Another possibility is to use a non-meridian instrument working in normal mode, such as the USNO CCD $20 \mathrm{~cm}$ Astrograph. The results obtained with this instrument seem to be very rewarding (Zacharias 1997). However, the particularity of the drift scan mode lies in the fact that the length of the field in right ascension is just limited by the duration of the night. For example, the size of the fields observed with Bordeaux and Valinhos CCD meridian circles is typically from 3 to 7 square degrees, as compared with 1 square degree in the case of 
the USNO CCD Astrograph. This fact can be a substantial advantage for zones where the reference star density is low, for example outside the galactic plane. Indeed, the density of the Tycho catalogue is very irregular, and some fields are very poor in reference stars, i.e. from 0 to 5 Tycho stars per square degree, and with a very inhomogeneous distribution in the field (ESA 1997d). In such a configuration, reducing the field to 1 square degree may be very problematic, but to take exposures with overlap could help to solve this problem. Another fact which can help to decide between both configurations is the elimination of fluctuations due to the atmosphere. Drift scan or otherwise? We need a few years more to prove superiority of one method over the other.

Acknowledgements. The authors are grateful to N. Ralite and M. Tellechea for their participation in the observations made in Bordeaux, as well as to all the persons who participated in the observations made at Valinhos. The authors also wish to thank C. Ducourant, M. Odenkirchen and C. Soubiran for constructive discussions for the drafting of this paper. This work was supported by the CNRS (URA 352) and the Aquitaine Region in France, and by the CNPq and the FAPESP in Brazil. B. Viateau was supported by the French CNES through a postdoctoral grant.

\section{References}

Benevides-Soares P., Teixeira R., 1992, A\&A 253, 307

Benevides-Soares P., Teixeira R., Réquième Y., 1993, A\&A 278, 293

Eichhorn H., 1960, Astron. Nachr. 285, 233

ESA, 1997a, The Hipparcos and Tycho Catalogues, volume 3, ESA SP-1200

ESA, 1997b, The Hipparcos and Tycho Catalogues, volume 4, ESA SP-1200, p. 220

ESA, 1997c, The Hipparcos and Tycho Catalogues, volume 4,
ESA SP-1200, p. 262

ESA, 1997d, The Hipparcos and Tycho catalogues, volumes 14-16, ESA SP-1200

Froeschlé M., 1998 (private communication)

Høg E., 1968, Zeitschrift für Astrophysik 69, 313

Lasker B.M., Jenkner H., Russell J.L., 1988, IAU Symp. 133, Débarbat S. et al. (eds.), p. 229

Lazorenko P.F., 1992, Kinematika i Fiziea Nebesnik Tel. 8, 3, p. 78

Lépine J.R.D., Duvert G., 1994, A\&A 286, 60

Morrison L.V., 1985-94, Carlsberg Meridian Catalogues Numbers 1-8, Copenhagen University Observatory, Royal Greewich Observatory, Real Instituto y Observatorio de la Marina en San Fernando

Odenkirchen M., Soubiran C., Le Campion J.F., 1998, "Early epoch stellar positions from the Bordeaux Carte du Ciel", Proceedings of the International Spring Meeting of the Astronomische Gesellschaft, Gotha, Germany (to be published)

Rapaport M., Le Campion J.F., 1990, "Convergence de méthodes itératives utilisées en astrométrie", Proceedings of the Journées 1990 Systèmes de référence spatio-temporels, Paris, France, p. 75

Réquième Y., Le Campion J.F., Montignac G., et al., 1997, in: Hipparcos Venice'97, ESA SP-402, p. 135

Stone R.C., 1993, in: Developments in Astrometry and Astrophysics and their impact on Astrophysics and Geodynamics, IAU Symposium 156, Mueller I.I. and Kolaczek B. (eds.). Kluwer, Dordrecht, p. 65

Stone R.C., Monet D.G., Monet A.K., et al., 1996, AJ 111, 1721

Teixeira R., Réquième Y., Benevides-Soares P., Rapaport M., 1992, A\&A 264, 307

Urban S.E., Corbin T.E., Wycoff G.L., et al., 1998a, AJ 115, 1212

Urban S.E., Corbin T.E., Wycoff G.L., 1998b, AJ 115, 2161

Viateau B., Rapaport M., 1998, A\&A 334, 729

Wendker H.J., 1995, A\&AS 109, 177

Zacharias N., 1997, AJ 113, 1925 